\title{
Erratum
}

\section{Product Integration for the Linear Transport Equation in Slab Geometry}

G. Monegato ${ }^{1}$ and V. Colombo ${ }^{2}$

1 Dipartimento di Matematica, Politecnico di Torino, Italy

2 Dipartimento di Energetica, Politecnico di Torino, Italy

On page 230 the polynomial $t_{n}(u)$ should be chosen such that in $[-1,1]$

$$
\left(1-u^{2}\right)^{2}\left|f(u)-t_{n}(u)\right| \leq \text { const } \cdot\left(\frac{\sqrt{1-u^{2}}}{n}\right)^{3-\delta}, \delta>0 \quad \text { small as we like. }
$$

The existence of such a polynomial is guarantee by Theorem 4.2 in [1] applied to the function $\left(1-u^{2}\right)^{2} f(u)$.

Then, the analysis of $R_{n}^{(2)}(f ; s)$ for our kernels $k(s ; u)$ leads to the bound

$$
\left\|R_{n}^{(2)}(f ; s)\right\|_{\infty}=o\left(n^{-3}\right),
$$

which is sufficient to prove our convergence result.

On page 231, lines $1^{-}$and $9^{-}$, replace $O\left(R^{k+\delta}\right)$ by $O\left(R^{j+\delta}\right)$ and $\rho=O\left(n^{-2}\right)$ by the sentence: $\rho^{\prime}$ sufficiently small.

\section{Reference}

1. Hinnemann, E., Gonska, H.H.: Generalization of a theorem of De Vore. In: Chui, C.K., Schumaker, L.L., Ward, J.D., (eds.) Approximation Theory IV, pp. 527-532. London New York: Academic Press 1983 